\title{
Holographic cosmology and tachyon inflation
}

\author{
Neven Bilić * \\ Division of Theoretical Physics, Rudjer Bošković Institute, \\ Zagreb, Croatia
}

March 1, 2022

\begin{abstract}
After a brief introduction to the AdS/CFT holography a tachyon inflation will be discussed in the framework of holographic cosmology. The model is based on a holographic braneworld scenario with an effective tachyon field on a D3-brane located at the holographic bound of an asymptotic $\mathrm{ADS}_{5}$ bulk.
\end{abstract}

\section{Introduction}

Branewarld cosmology is based on the scenario in which matter is confined on a brane moving in the higher dimensional bulk with only gravity allowed to propagate in the bulk [1, 2, 3, 4]. The brane can be placed, e.g., at the boundary of a 5-dim asymptotically anti de Sitter (AdS) space-time. We shall refer to this type of braneworld as the holographic braneworld [5, 6].

It is important to stress that AdS space appears naturally in the context of $\mathrm{M} /$ string theory. The point is that pure supergravity theories, without additional supersymmetric matter in arbitrary dimensions, necessarily satisfy the strong energy condition [7]. After compactification of 10 or 11-dimensional theory down to 4+1-dimensional spacetime $\mathrm{X}$, the stress tensor in $\mathrm{X}$ also satisfies the strong energy condition. As a consequence, if we wish $\mathrm{X}$ to be a maximally symmetric spacetime, it must be an Einstein space with non-positive cosmological constant, i.e., X must be either Minkowski or anti de Sitter.

In the study of the holographic braneworld a crucial property of an asymptotically AdS bulk is that AdS space is dual to a conformal field theory at its boundary. The so called anti de Sitter/conformal field theory (AdS/CFT) correspondence [8] reflects an obvious symmetry relationship: on the bulk side, $\mathrm{AdS}_{5}$ is a maximally symmetric solution to Einsteins equations with negative cosmological constant with the symmetry group $\mathrm{AdS}_{5} \equiv \mathrm{SO}(4,2)$; on the boundary side, the boundary conformal field theory is invariant under conformal transformations: Poincaré + dilatations + special conformal transformation. These transformations constitute the conformal group which is locally isomorphic to the $\mathrm{SO}(4,2)$ symmetry group.

\footnotetext{
*bilic@irb.hr
} 


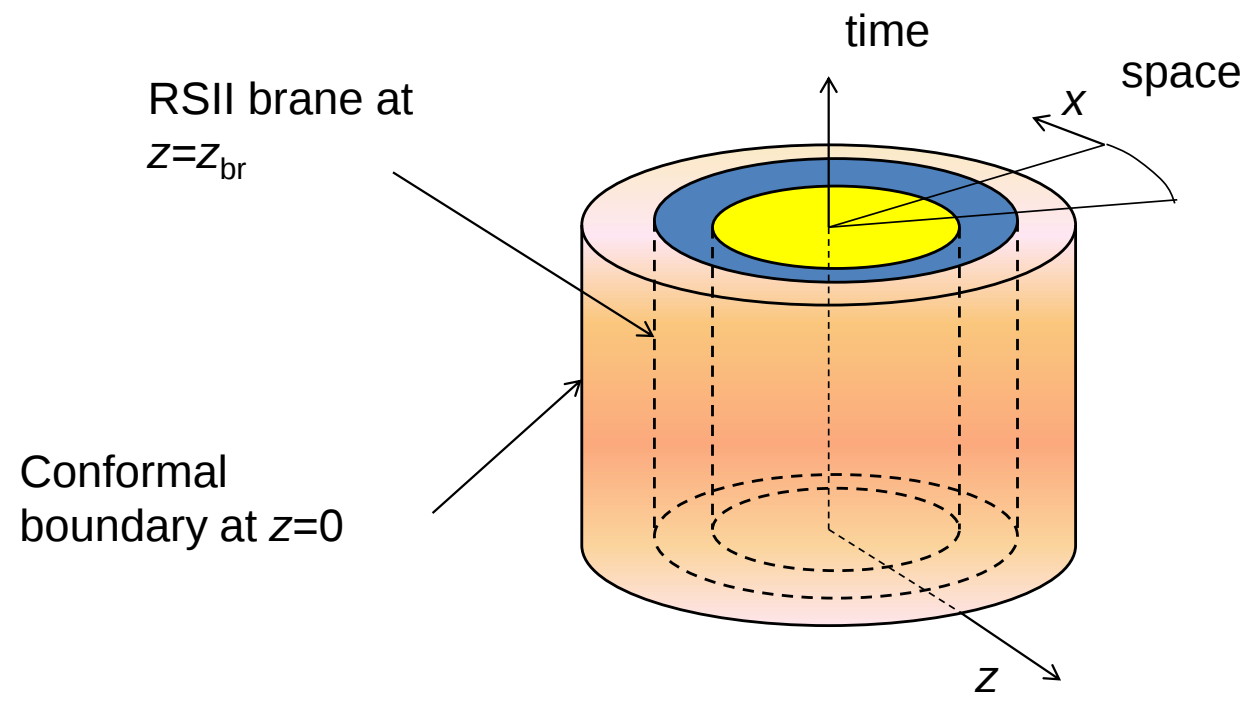

Figure 1: Illustration of the $\mathrm{AdS}_{5}$ bulk with two branes: Randall-Sundrum (RSII) brane located at $z=z_{\mathrm{br}}$ and the holographic brane at $z=0$.

Our aim here is to study a model of tachyon inflation in the framework of holographic cosmology. In tachyon inflation models [9, 10, 11, 12, 13] inflation is driven by the tachyon field originating in string theory. In particular, our model is based on a holographic braneworld scenario with an effective tachyon field on the D3-brane located at the holographic boundary of an asymptotic $\mathrm{ADS}_{5}$ bulk. This scenario is partly based on our previous works [14, 15, 16].

The remainder of the paper is organized as follows. We begin by section 2 in which we give brief introduction to the AdS/CFT correspondence including the holographic renormalization (section 2.1) and a derivation of holographic cosmology (section 2.2). In this section we add a subsection 2.3 in which we comment on a modified Gauss-Bonnet gravity in relation to holographic cosmology. In section 3 we propose a model for tachyon inflation and present preliminary results. Conclusions and outlook are given in section 4 .

\section{AdS/CFT and holographic cosmology}

A general asymptotically $\mathrm{AdS}_{5}$ metric in Fefferman-Graham coordinates [17] is of the form

$$
d s^{2}=G_{a b} d x^{a} d x^{b}=\frac{\ell^{2}}{z^{2}}\left(g_{\mu \nu} d x^{\mu} d x^{\nu}-d z^{2}\right)
$$


where the length scale $\ell$ is the AdS curvature radius and we use the Latin alphabet for $4+1$ and the Greek alphabet for $3+1$ spacetime indices. Consider a 5-dim bulk action in $\mathrm{AdS}_{5}$ background

$$
S_{(5)}[\Phi]=\int d^{5} x \sqrt{G} \mathcal{L}_{(5)}\left(\Phi, G_{a b}\right)
$$

Given an induced metric $h_{\mu \nu}$ on the boundary and a boundary value $\Phi(x, z=0) \equiv \phi(x)$, the bulk field $\Phi$ is completely determined by its field equations obtained from the variational principle

$$
\frac{\delta S_{(5)}}{\delta \Phi}=0
$$

A solution $\Phi[\phi, h]$ to this equation can be used to define a functional

$$
S[\phi, h]=S_{(5)}^{\mathrm{sh}}[\Phi[\phi, h]]
$$

where $S_{(5)}^{\mathrm{sh}}[\Phi[\phi, h]]$ is the on shell bulk action. Then the AdS/CFT conjecture states that $S[\phi, h]$ can be identified with the generating functional of a conformal field theory (CFT) on the boundary, i.e.,

$$
S[\phi, h] \equiv \ln \int d \psi \exp \left\{-\int d^{4} x \sqrt{|h|}\left[\mathcal{L}^{\mathrm{CFT}}(\psi(x))-O(\psi(x)) \phi(x)\right]\right\},
$$

where $\mathcal{L}^{\mathrm{CFT}}$ is a CFT Lagrangian, $O(\psi)$ are CFT operators of dimension $\Delta$, and the boundary fields serve as sources for CFT operators. In this way the CFT correlation functions can be calculated as functional derivatives of the on-shell bulk action, e.g.,

$$
\frac{\delta^{2} S_{(5)}}{\delta \phi(x) \delta \phi(y)}=\langle O(\psi(x)) O(\psi(y))\rangle-\langle O(\psi(x))\rangle\langle O(\psi(y))\rangle .
$$

Similarly, the induced metric $h_{\mu \nu}$ serves as the source for the the stress tensor operator of the dual CFT so that its vacuum expectation value is obtained as

$$
\frac{1}{2 \sqrt{|h|}} \frac{\delta S_{(5)}}{\delta h^{\mu \nu}}=\left\langle T_{\mu \nu}^{\mathrm{CFT}}\right\rangle
$$

\subsection{Holographic renormalization}

Consider next the bulk action with only gravity in the bulk

$$
S_{(5)}[G]=\frac{1}{8 \pi G_{5}} \int d^{5} x \sqrt{G}\left[-\frac{R^{(5)}[G]}{2}-\Lambda_{5}\right],
$$

where $G_{5}$ is the five-dimensional gravitational constant and $\Lambda_{5}$ is the bulk cosmological constant related to the AdS curvature radius as $\Lambda_{5}=-6 / \ell^{2}$. The on-shell action is IR divergent and must be regularized and renormalized. The asymptotically AdS metric near $z=0$ can be expanded as

$$
g_{\mu \nu}(z, x)=g_{\mu \nu}^{(0)}(x)+z^{2} g_{\mu \nu}^{(2)}(x)+z^{4} g_{\mu \nu}^{(4)}(x)+\cdots
$$


Explicit expressions for $g_{\mu \nu}^{(2 n)}$ in terms of arbitrary $g_{\mu \nu}^{(0)}$ can be found in Ref. [18]. We regularize the action by placing a brane (RSII brane) near the boundary, i.e., at $z=\epsilon \ell, \epsilon \ll 1$, so that the induced metric on the brane is

$$
h_{\mu \nu}=\frac{1}{\epsilon^{2}} g_{\mu \nu}(\epsilon \ell, x)=\frac{1}{\epsilon^{2}}\left(g_{\mu \nu}^{(0)}+\epsilon^{2} \ell^{2} g_{\mu \nu}^{(2)}+\epsilon^{4} \ell^{4} g_{\mu \nu}^{(4)}+\cdots\right) \text {. }
$$

The bulk splits in two regions: $0 \leq z<\epsilon \ell$ and $\epsilon \ell \leq z<\infty$. We can either discard the $0 \leq z<\epsilon \ell$ region (one-sided regularization) or invoke the $\mathrm{Z}_{2}$ symmetry and identify two regions (two-sided regularization). For simplicity we shall use the one-sided regularization. The regularized on shell bulk action is [19]

$$
S_{(5)}^{\mathrm{reg}}[h]=\frac{1}{8 \pi G_{5}} \int_{z \geq \epsilon \ell} d^{5} x \sqrt{G}\left[-\frac{R^{(5)}}{2}-\Lambda_{5}\right]+S_{\mathrm{GH}}[h]+S_{\mathrm{br}}[h],
$$

where $S_{\mathrm{GH}}$ is the Gibbons-Hawking boundary term and the brane action is given by

$$
S_{\mathrm{br}}[h]=\int d^{4} x \sqrt{|h|}\left(-\sigma+\mathcal{L}_{\text {matt }}[h]\right),
$$

where $\sigma$ is the brane tension and the Lagrangian $\mathcal{L}_{\text {matt }}$ describes matter on the brane. The renormalized action is obtained by adding counter-terms and taking the limit $\epsilon \rightarrow 0$

$$
S^{\mathrm{ren}}[h]=S_{(5)}^{\mathrm{reg}}[h]+S_{1}[h]+S_{2}[h]+S_{3}[h]
$$

The necessary counter-terms are [18, 20]

$$
\begin{gathered}
S_{1}[h]=-\frac{6}{16 \pi G_{5} \ell} \int d^{4} x \sqrt{|h|} \\
S_{2}[h]=-\frac{\ell}{16 \pi G_{5}} \int d^{4} x \sqrt{|h|}\left(-\frac{R[h]}{2}\right), \\
S_{3}[h]=-\frac{\ell^{3}}{16 \pi G_{5}} \int d^{4} x \sqrt{|h|} \frac{\log \epsilon}{4}\left(R^{\mu \nu}[h] R_{\mu \nu}[h]-\frac{1}{3} R^{2}[h]\right) .
\end{gathered}
$$

The last term is scheme dependent and its integrand is proportional to the holographic conformal anomaly [21]. Now we demand that the variation with respect to the induced metric $h_{\mu \nu}$ of the regularized on shell bulk action (RSII action) vanishes, i.e., we require

$$
\delta S_{(5)}^{\mathrm{reg}}[h]=0
$$

which may be expressed as

$$
\begin{aligned}
& \delta\left[S^{\text {ren }}-S_{3}-\left(\sigma-\frac{3}{8 \pi G_{5} \ell}\right) \int d^{4} x \sqrt{|h|}+\int d^{4} x \sqrt{|h|} \mathcal{L}_{\text {matt }}\right. \\
& \left.-\frac{\ell}{16 \pi G_{5}} \int d^{4} x \sqrt{|h|} \frac{R[h]}{2}\right]=0 .
\end{aligned}
$$


The third term gives the contribution to the cosmological constant and may be eliminated by imposing the RSII fine-tuning condition

$$
\sigma=\frac{3}{8 \pi G_{5} \ell} .
$$

The variation of the scheme dependent $S_{3}$ may be combined with the first term so that

$$
\frac{2}{\sqrt{-h}} \frac{\delta S^{\mathrm{ren}}}{\delta h^{\mu \nu}}-\frac{2}{\sqrt{-h}} \frac{\delta S_{3}}{\delta h^{\mu \nu}}=\left\langle T_{\mu \nu}^{\mathrm{CFT}}\right\rangle
$$

according to the AdS/CFT prescription (7). The net effect of $\delta S_{3}$ is that it cancels the $\square R$ term in the conformal anomaly [22] so the trace of the CFT stress tensor simply reads

$$
\left\langle T_{\mu}^{\mathrm{CFT} \mu}\right\rangle=-\frac{\ell^{3}}{64 \pi G_{5}}\left(R^{\mu \nu} R_{\mu \nu}-\frac{1}{3} R^{2}\right) .
$$

The variation equation (18) yields four-dimensional Einstein's equations on the boundary

$$
R_{\mu \nu}-\frac{1}{2} R g_{\mu \nu}^{(0)}=8 \pi G_{\mathrm{N}}\left(\left\langle T_{\mu \nu}^{\mathrm{CFT}}\right\rangle+T_{\mu \nu}^{\mathrm{matt}}\right),
$$

where $R_{\mu \nu}$ is the Ricci tensor associated with the metric $g_{\mu \nu}^{(0)}$ and the energy energy-momentum tensor

$$
T_{\nu}^{\operatorname{matt} \mu}=\operatorname{diag}(\rho,-p,-p,-p)
$$

corresponds to the Lagrangian $\mathcal{L}_{\text {matt }}$ and describes matter on the brane. Thanks to the AdS/CFT correspondence, the vacuum expectation value $\left\langle T_{\mu \nu}^{\mathrm{CFT}}\right\rangle$ is obtained in terms of quantities related to the bulk metric [18]

$$
\left\langle T_{\mu \nu}^{\mathrm{CFT}}\right\rangle=\frac{\ell^{3}}{4 \pi G_{5}}\left\{\frac{1}{8}\left[\left(\operatorname{Tr} g^{(2)}\right)^{2}-\operatorname{Tr}\left(g^{(2)}\right)^{2}\right] g_{\mu \nu}^{(0)}+\frac{1}{2}\left(g^{(2)}\right)_{\mu \nu}^{2}-\frac{1}{4} \operatorname{Tr} g^{(2)} g_{\mu \nu}^{(2)}-g_{\mu \nu}^{(4)}\right\} .
$$

In the next section we will specify the boundary metric in the FRW form and derive the evolution equations on the holographic brane.

\subsection{Holographic cosmology}

Here we outline a derivation of the Friedmann equations on the holographic brane following Refs. [5, 6]. For this purpose it is convenient to represent the bulk metric in AdSSchwarzschild static coordinates [23]

$$
d s_{(5)}^{2}=f(r) d \tau^{2}-\frac{d r^{2}}{f(r)}-r^{2} d \Omega_{k}^{2}
$$

where

$$
f(r)=\frac{r^{2}}{\ell^{2}}+k-\mu \frac{\ell^{2}}{r^{2}}
$$

and

$$
d \Omega_{k}^{2}=d \chi^{2}+\frac{\sin ^{2}(\sqrt{k} \chi)}{k}\left(d \vartheta^{2}+\sin ^{2} \vartheta d \varphi^{2}\right)
$$


is the spatial line element for a closed $(k=1)$, open hyperbolic $(k=-1)$, or open flat $(k=0)$ space. The dimensionless parameter $\mu$ is related to the black-hole mass via [24, 25]

$$
\mu=\frac{8 G_{5} M_{\mathrm{bh}}}{3 \pi \ell^{2}}
$$

Starting from (25) we make a coordinate transformation

$$
\tau=\tau(t, z), \quad r=r(t, z)
$$

such that the line element in new coordinates takes the form

$$
d s_{(5)}^{2}=\frac{\ell^{2}}{z^{2}}\left(\mathcal{N}^{2}(t, z) d t^{2}-\mathcal{A}^{2}(t, z) d \Omega_{k}^{2}-d z^{2}\right)
$$

where

$$
\mathcal{A}^{2}(t, z)=\frac{z^{2}}{\ell^{2}} r^{2}(t, z)
$$

and $\mathcal{N}$ is fixed by the requirement that the off diagonal elements of the transformed metric vanish. Next, imposing the boundary conditions at $\mathrm{z}=0$ :

$$
\mathcal{N}(t, 0)=1, \quad \mathcal{A}(t, 0)=a(t),
$$

we obtain the induced metric at the boundary in the FRW form

$$
d s^{2}=g_{\mu \nu}^{(0)} d x^{\mu} d x^{\nu}=d t^{2}-a^{2}(t) d \Omega_{k}^{2} .
$$

Solving Einsteins equations in the bulk one finds [5]

$$
\begin{gathered}
\mathcal{A}^{2}=a^{2}\left[1-\left(H^{2}+\frac{k}{a^{2}}\right) \frac{z^{2}}{4}\right]^{2}+\frac{1}{4} \frac{\mu z^{4}}{a^{2}}, \\
\mathcal{N}=\frac{\dot{\mathcal{A}}}{\dot{a}},
\end{gathered}
$$

where $H=\dot{a} / a$ is the Hubble expansion rate on the $z=0$ boundary. Comparing the exact solution with the expansion (9) we can extract $g_{\mu \nu}^{(2)}$ and $g_{\mu \nu}^{(4)}$. Then, using (24) we find the expression for $T^{\mathrm{CFT}}$ in the special case of the boundary metric (33)

$$
\left\langle T_{\mu \nu}^{\mathrm{CFT}}\right\rangle=t_{\mu \nu}+\frac{1}{4}\left\langle T_{\alpha}^{\mathrm{CFT} \alpha}\right\rangle g_{\mu \nu}^{(0)}
$$

The second term on the right-hand side corresponds to the conformal anomaly

$$
\left\langle T_{\alpha}^{\mathrm{CFT} \alpha}\right\rangle=\frac{3 \ell^{3}}{16 \pi G_{5}} \frac{\ddot{a}}{a}\left(H^{2}+\frac{k}{a^{2}}\right) .
$$

The first term on the right-hand side of (36) is a traceless tensor the nonvanishing components of which are

$$
t_{00}=-3 t_{i}^{i}=\frac{3 \ell^{3}}{64 \pi G_{5}}\left[\left(H^{2}+\frac{k}{a^{2}}\right)^{2}+\frac{4 \mu}{a^{4}}-\frac{\ddot{a}}{\dot{a}}\left(H^{2}+\frac{k}{a^{2}}\right)\right]
$$


Hence, apart from the conformal anomaly, the CFT dual to the time dependent asymptotically $\mathrm{AdS}_{5}$ metric (30) is a conformal fluid with the equation of state $p_{\mathrm{CFT}}=\rho_{\mathrm{CFT}} / 3$, where $\rho_{\mathrm{CFT}}=t_{00}, p_{\mathrm{CFT}}=-t_{i}^{i}$.

Next, using the effective Einstein equations (22) we obtain the holographic Friedmann equation [5, 22] which for a spatially flat geometry $(k=0)$ takes the form

$$
H^{2}-\frac{\ell^{2}}{4} H^{4}=\frac{8 \pi G_{\mathrm{N}}}{3} \rho+\frac{4 \mu}{a^{4}}
$$

Hence, one finds a deviation from the standard cosmology in two terms: the term proportional to $\mathrm{H}^{4}$ on the left-hand side and the last term on the right-hand side, the so called "dark radiation". Combining (39) and the energy conservation equation

$$
\dot{\rho}+3 H(p+\rho)=0
$$

one can easily derive the second Friedmann equation on the holographic brane

$$
\dot{H}\left(1-\frac{\ell^{2}}{2} H^{2}\right)=-4 \pi G_{\mathrm{N}}(p+\rho) .
$$

It is worth mentioning that holographic type cosmologies have been studied in other contexts [26, 27, 28, 29, 30, 31]. In particular, a modified Gauss-Bonnett gravity [32] in a cosmological context leads in a special case to the equation of the form (39) with $\mu=0$. It is instructive to briefly elaborate on this special form of modified Gauss-Bonnet gravity following C. Gao [29].

\subsection{Connection with modified Gauss-Bonnet gravity}

A modified Gauss-Bonnet gravity belongs to a class of modified gravity models in which the gravitational action is a general function of two variables: the Ricci scalar $R$ and the Gauss-Bonnet invariant

$$
\mathcal{G}=R^{2}-4 R^{\mu \nu} R_{\mu \nu}+R^{\mu \nu \rho \sigma} R_{\mu \nu \rho \sigma} .
$$

The total action in such a model can be written in the form

$$
S=\int d^{4} x \sqrt{-g}\left[\frac{1}{16 \pi G_{\mathrm{N}}}(-R+f(R, \mathcal{G}))+\mathcal{L}_{\text {matt }}\right],
$$

where $f$ is a smooth function. This class of models was shown to be ghost free [33, 34. In a cosmological context it is natural to require in addition that the second Friedmann equation is linear in $\dot{H}$. Then it follows [29] that $f$ must be a function of only one variable $f=f(J)$ where

$$
J=\frac{1}{\sqrt{12}}\left(-R+\sqrt{R^{2}-6 \mathcal{G}}\right)^{1 / 2}
$$

For a spatially flat metric one finds $J=\dot{a} / a \equiv H$ and the first Friedmann equation takes the form

$$
H^{2}+\frac{1}{6}\left(f(H)-H \frac{d f}{d H}\right)=\frac{8 \pi G_{\mathrm{N}}}{3} \rho
$$


Hence, the left hand side is a function of $H$ only and the second Friedmann equation will be linear in $\dot{H}$. In particular, for

$$
f=\frac{1}{2} \ell^{2} H^{4}
$$

the Friedman equation takes the holographic form (39) with $\mu=0$. Thus, the holographic cosmology is reproduced in a modified Gauss-Bonnet gravity with action

$$
S=\int d^{4} x \sqrt{-g}\left[\frac{1}{16 \pi G_{\mathrm{N}}}\left(-R+\frac{\ell^{2}}{288}\left(\sqrt{R^{2}-6 \mathcal{G}}-R\right)^{2}\right)+\mathcal{L}_{\text {matt }}\right] .
$$

\section{Tachyon inflation on the holographic brane}

Here we give a brief recapitulation of the results which will be presented in more detail elsewhere [35]. We start from a general tachyon Lagrangian

$$
\mathcal{L}=-\ell^{-4} V(\theta / \ell) \sqrt{1-g^{\mu \nu} \theta_{, \mu} \theta_{, \nu}},
$$

where the field $\theta$ is of dimension of length and the $\mathrm{AdS}_{5}$ curvature radius $\ell$ fixes the scale. The potential $V$ is an arbitrary non-increasing function. Assuming a spatially flat FRW metric on the holographic brane we solve the field equations augmented by the holographic Friedman equation (39) with $\mu=0$,

$$
H^{2}-\frac{\ell^{2}}{4} H^{4}=\frac{8 \pi G_{\mathrm{N}}}{3} \rho,
$$

where $\rho=\ell^{-4} V / \sqrt{1-\dot{\theta}^{2}}$. Solving (49) as a quadratic equation for $H^{2}$ we find

$$
H^{2}=\frac{2}{\ell^{2}}\left(1 \pm \sqrt{1-\frac{8 \pi G_{\mathrm{N}}}{3} \ell^{2} \rho}\right)
$$

Demanding that this equation reduces to the standard Friedmann equation in the low density limit, i.e., in the limit when $G_{\mathrm{N}} \ell^{2} \rho \ll 1$, we can discard the $(+)$ sign solution. Then from (50) it follows that $H^{2}$ can vary between zero and its maximal value $H_{\max }^{2}=2 / \ell^{2}$ corresponding to the maximal energy density $\rho_{\max }=3 /\left(8 \pi G \ell^{2}\right)$ [6, 36]. If in addition we assume no violation of the weak energy condition $\rho \geq 0, p+\rho \geq 0$, the expansion rate will, according to (41), be a monotonously decreasing function of time. The universe evolution starts from $t=0$ with an initial $H_{\mathrm{i}} \leq H_{\text {max }}$ with energy density and cosmological scale both finite. Hence, as already noted in Ref. [29], in the modified cosmology described by the Friedmann equation (49) the Big Bang singularity is avoided!

In the following we will examine a simple exponential potential $V=e^{-\omega x}$ where $\omega$ is a free dimensionless parameter. This potential has been extensively exploited in the tachyon literature [11, 12, 13, 37].

Tachyon inflation is based upon the slow evolution of $\theta$ with the slow-roll conditions

$$
\dot{\theta}^{2} \ll 1, \quad|\ddot{\theta}| \ll 3 H \dot{\theta}
$$




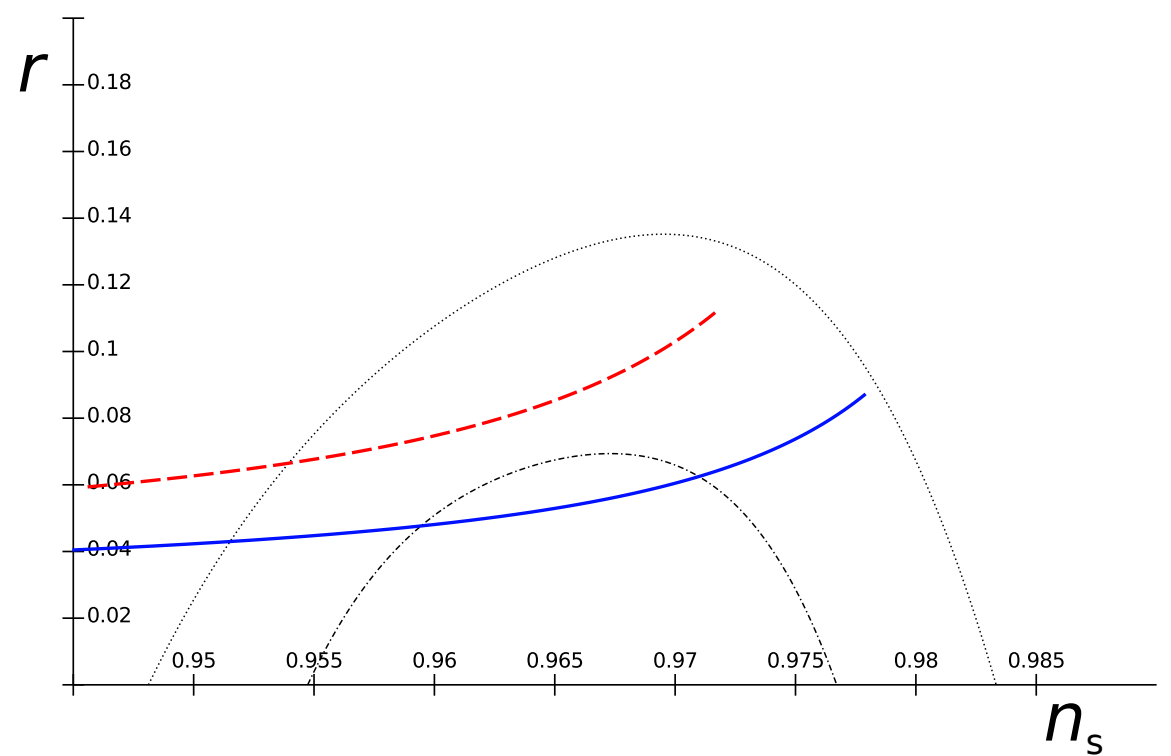

Figure 2: $r$ versus $n_{\mathrm{s}}$ for fixed $N=70$ (dashed red line) and $N=90$ (full blue line) and varying initial values $H_{i}^{2}$ ranging from 0 to $2 \ell^{-2}$ along the lines. The parameter $\omega$ is also varying in view of (54). The dash-dotted and dotted lines denote the Planck contours of the one and two $\sigma$ constraints, respectively.

Then, during inflation we find

$$
H^{2} \ell^{2} \simeq 2\left(1-\sqrt{1-\kappa^{2} V / 3}\right),
$$

where $\kappa^{2}=8 \pi G / \ell^{2}$. Using this we can calculate the so called number of e-folds defined as

$$
N \equiv \int_{t_{\mathrm{i}}}^{t_{\mathrm{f}}} H d t
$$

where the subscripts $i$ and $f$ denote respectively the beginning and the end of inflation. This can be calculated explicitly yielding an expression that relates the initial expansion rate $H_{\mathrm{i}}$ to $\omega$ and $N$

$$
N=\frac{12}{\omega^{2}}\left[\sqrt{1-\frac{\omega^{2}}{3}}-1+\frac{H_{\mathrm{i}}^{2} \ell^{2}}{2}+\ln \left(2-\frac{H_{\mathrm{i}}^{2} \ell^{2}}{2}\right)-\ln \left(1+\sqrt{1-\frac{\omega^{2}}{3}}\right)\right] .
$$

The slow-roll inflation parameters

$$
\varepsilon_{1} \equiv-\frac{\dot{H}}{H^{2}}=\frac{\omega^{2}\left(4-H^{2} \ell^{2}\right)}{6 H^{2} \ell^{2}\left(2-H^{2} \ell^{2}\right)}, \quad \varepsilon_{2} \equiv \frac{\dot{\varepsilon_{1}}}{H \varepsilon_{1}}=2 \varepsilon_{1}\left(1-\frac{2 H^{2} \ell^{2}}{\left(2-H^{2} \ell^{2}\right)\left(4-H^{2} \ell^{2}\right)}\right),
$$

are related to the tensor-to-scalar ratio $r$ and the scalar spectral index $n_{\mathrm{s}}$ defined by

$$
r=\frac{\mathcal{P}_{\mathrm{T}}}{\mathcal{P}_{\mathrm{S}}}, \quad n_{\mathrm{s}}=\frac{d \ln \mathcal{P}_{\mathrm{S}}}{d \ln q},
$$


where $\mathcal{P}_{\mathrm{S}}$ and $\mathcal{P}_{\mathrm{T}}$ are the power spectra of scalar and tensor perturbations, respectively, evaluated at the horizon, i.e., for a wave-number satisfying $q=a H$. Combining the previously developed techniques for calculating the power spectra [10, 13, 36, 38] applied to the tachyon fluid we find at the lowest order in $\varepsilon_{1}$ and $\varepsilon_{2}$

$$
\begin{gathered}
r=16 \varepsilon_{1}\left(1-\frac{H^{2} \ell^{2}}{2}\right)\left(1-C \varepsilon_{2}-\frac{4-2 H^{2} \ell^{2}}{12-3 H^{2} \ell^{2}} \varepsilon_{1}\right), \\
n_{\mathrm{s}}=1+\left(-3+\frac{2-3 H^{2} \ell^{2}}{2-H^{2} \ell^{2}}\right) \varepsilon_{1}-\varepsilon_{2},
\end{gathered}
$$

where $C=-2+\ln 2+\gamma \simeq-0.72$ and $\gamma$ is the Euler constant. These expressions substantially deviate from the lowest order results in the canonical scalar inflation and standard tachyon inflation [13]. A comparison of our preliminary results with Planck 2015 [39] data (Fig. 2) as well as with the most recent Planck datal [40] shows a reasonable agreement.

\section{Conclusions and outlook}

In this brief review we have discussed a holographic cosmology in a braneworld scenario applied to tachyon inflation. We have shown that the slow-roll equations of the tachyon inflation with exponentially attenuating potential on the holographic brane are quite distinct from those of the standard tachyon inflation with the same potential. The presented results obtained in the slow roll approximation are preliminary. What remains to be done is to solve the exact equations numerically for the same potential and for various other potentials that have been exploited in the literature.

\section{Acknowledgments}

This work has been supported by the H2020 CSA Twinning project No. 692194, "RBI-TWINNING" and partially supported by the ICTP - SEENET-MTP project NT-03 Cosmology - Classical and Quantum Challenges and by the STSM CANTATA-COST grant. The author acknowledges hospitality of the Physics Department, University of Nis, Serbia.

\section{References}

[1] N. Arkani-Hamed, S. Dimopoulos, and G. Dvali, Phys. Lett. B 429, 263 (1998).

[2] I. Antoniadis, N. Arkani-Hamed, S. Dimopoulos and G. R. Dvali, Phys. Lett. B 436, 257 (1998) [hep-ph/9804398].

[3] L. Randall and R. Sundrum, Phys. Rev. Lett. 83, 3370 (1999)

[4] L. Randall and R. Sundrum, Phys. Rev. Lett. 83, 4690 (1999)

\footnotetext{
${ }^{1}$ The Planck 2018 data were released after the delivery of this talk.
} 
[5] P. S. Apostolopoulos, G. Siopsis and N. Tetradis, Phys. Rev. Lett. 102, 151301 (2009) arXiv:0809.3505 [hep-th]].

[6] N. Bilić, Phys. Rev. D 93, no. 6, 066010 (2016) arXiv:1511.07323 [gr-qc]].

[7] G. W. Gibbons, Class. Quant. Grav. 20, S321 (2003) hep-th/0301117.

[8] J.M. Maldacena, Adv. Theor. Math. Phys. 2, 231 (1998); Int. J. Theor. Phys. 38, 1113 (1999) [arXiv:hepth/9711200].

[9] M. Fairbairn and M. H. G. Tytgat, Phys. Lett. B 546, 1 (2002) hep-th/0204070; A. Feinstein, Phys. Rev. D 66, 063511 (2002) [hep-th/0204140]; G. Shiu and I. Wasserman, Phys. Lett. B 541, 6 (2002) hep-th/0205003]; L. Kofman and A. D. Linde, JHEP 0207, 004 (2002) [hep-th/0205121].

[10] A. V. Frolov, L. Kofman and A. A. Starobinsky, Phys. Lett. B 545, 8 (2002) hep-th/0204187;

[11] M. Sami, P. Chingangbam and T. Qureshi, Phys. Rev. D 66, 043530 (2002) hep-th/0205179]; G. Shiu, S. H. H. Tye and I. Wasserman, Phys. Rev. D 67, 083517 (2003) [hep-th/0207119]; P. Chingangbam, S. Panda and A. Deshamukhya, JHEP 0502, 052 (2005) |hep-th/0411210|; S. del Campo, R. Herrera and A. Toloza, Phys. Rev. D 79, 083507 (2009) arXiv:0904.1032] S. Li and A. R. Liddle, JCAP 1403, 044 (2014) arXiv:1311.4664.

[12] J. M. Cline, H. Firouzjahi and P. Martineau, JHEP 0211, 041 (2002) hep-th/0207156.

[13] D. A. Steer and F. Vernizzi, Phys. Rev. D 70, 043527 (2004) hep-th/0310139.

[14] N. Bilić, D. D. Dimitrijevic, G. S. Djordjevic and M. Milosevic, Int. J. Mod. Phys. A 32, no. 05, 1750039 (2017) arXiv:1607.04524 [gr-qc]].

[15] N. Bilić, S. Domazet and G. S. Djordjevic, Phys. Rev. D 96, no. 8, 083518 (2017) arXiv:1707.06023 [hep-th]]

[16] N. Bilić, S. Domazet and G. S. Djordjevic, Class. Quant. Grav. 34, no. 16, 165006 (2017) arXiv:1704.01072 [gr-qc]].

[17] C. Fefferman and C. R. Graham, arXiv:0710.0919 [math.DG]

[18] S. de Haro, S. N. Solodukhin and K. Skenderis, Commun. Math. Phys. 217, 595 (2001) hep-th/0002230.

[19] S. de Haro, K. Skenderis and S. N. Solodukhin, Class. Quant. Grav. 18, 3171 (2001) hep-th/0011230.

[20] S. W. Hawking, T. Hertog and H. S. Reall, Phys. Rev. D 62, 043501 (2000) hep-th/0003052.

[21] M. Henningson and K. Skenderis, JHEP 9807, 023 (1998) hep-th/9806087. 
[22] E. Kiritsis, JCAP 0510, 014 (2005) hep-th/0504219].

[23] D. Birmingham, Class. Quant. Grav. 16, 1197 (1999) hep-th/9808032.

[24] R. C. Myers and M. J. Perry, Annals Phys. 172, 304 (1986).

[25] E. Witten, Adv. Theor. Math. Phys. 2, 505 (1998) [hep-th/9803131].

[26] A. O. Barvinsky, C. Deffayet and A. Y. Kamenshchik, JCAP 0805, 020 (2008) arXiv:0801.2063 [hep-th]].

[27] J. E. Lidsey, Phys. Rev. D 88, 103519 (2013) [arXiv:0911.3286 [hep-th]].

[28] S. Viaggiu, Mod. Phys. Lett. A 31, no. 04, 1650016 (2016) [arXiv:1511.06511 [hep-th]].

[29] C. Gao, Phys. Rev. D 86, 103512 (2012) [arXiv:1208.2790 [gr-qc]].

[30] E. Kiritsis, JCAP 1311, 011 (2013) [arXiv:1307.5873 [hep-th]].

[31] P. Binetruy, E. Kiritsis, J. Mabillard, M. Pieroni and C. Rosset, JCAP 1504, no. 04, 033 (2015) arXiv:1407.0820 [astro-ph.CO]].

[32] G. Cognola, E. Elizalde, S. Nojiri, S. D. Odintsov and S. Zerbini, Phys. Rev. D 73, 084007 (2006) [hep-th/0601008].

[33] D. Comelli, Phys. Rev. D 72, 064018 (2005) gr-qc/0505088.

[34] I. Navarro and K. Van Acoleyen, Phys. Lett. B 622, 1 (2005) [gr-qc/0506096].

[35] N. Bilić, D. D. Dimitrijevic, G. S. Djordjevic, M. Milosevic, and M. Stojanovic, work in preparation.

[36] S. del Campo, JCAP 1212, 005 (2012) arXiv:1212.1315 [astro-ph.CO]].

[37] A. Sen, Mod. Phys. Lett. A 17, 1797 (2002) hep-th/0204143.

[38] J. c. Hwang and H. Noh, Phys. Rev. D 66, 084009 (2002) hep-th/0206100].

[39] P. A. R. Ade et al. [Planck Collaboration], Astron. Astrophys. 594, A13 (2016) arXiv:1502.01589 [astro-ph.CO]].

[40] N. Aghanim et al. [Planck Collaboration], arXiv:1807.06209 [astro-ph.CO]; Y. Akrami et al. [Planck Collaboration], arXiv:1807.06211 [astro-ph.CO]. 